\title{
NONDEGENERACY FOR LIE TRIPLE SYSTEMS AND KANTOR PAIRS
}

\author{
ESTHER GARCÍA, MIGUEL GÓMEZ LOZANO, AND ERHARD NEHER
}

Dedicated to the memory of Issai Kantor (1936-2006)

\begin{abstract}
We study the transfer of nondegeneracy between Lie triple systems and their standard Lie algebra envelopes as well as between Kantor pairs, their associated Lie triple systems and their Lie algebra envelopes. We also show that simple Kantor pairs and Lie triple systems in characteristic 0 are nondegenerate.
\end{abstract}

\section{INTRODUCTION}

Kantor pairs are generalizations of Jordan pairs: The symmetry of the Jordan triple product in the outer variables is replaced by some weaker condition (1.5). This looks like a minor generalization. It is therefore all the more surprising that there are few general published results on Kantor pairs, despite a rather elaborate structure theory of Jordan pairs. For example, there does not seem to exist a list of all simple Kantor pairs.

The motivation of this paper is to initiate the structure theory of Kantor pairs. Our reason for doing so goes beyond a pure generalization for the sake of generalization. Rather, we expect that Kantor pairs will play an important rôle in a general structure theory of Lie algebras. The reason for this is the close connection between Kantor pairs and Lie algebras.

An example of a Kantor pair is the pair $V=\left(L_{1}, L_{-1}\right)$ obtained from a Lie algebra $L$ with a 5 -grading, i.e., a $\mathbb{Z}$-grading $L=L_{-2} \oplus L_{-1} \oplus L_{0} \oplus L_{1} \oplus L_{2}$, together with the triple products $\{\ldots\}: V^{\sigma} \times V^{-\sigma} \times V^{\sigma} \rightarrow V^{\sigma}, \sigma= \pm$, induced by the Lie algebra bracket $\{x y z\}=[[x, y], z]$. This is in fact not really an "example", since any Kantor pair arises in this way from a 5 -graded Lie algebra. There is a natural choice for such a Lie algebra, the so-called standard Lie envelope $\mathcal{L}_{V}$. Because of this close connection between Kantor pairs and Lie algebras, it is natural to study Kantor pairs $V$ via their Lie envelopes $\mathcal{L}_{V}$. Of course, this will only work if properties like simplicity, (semi)primeness or nondegeneracy of $V$ are reflected by

2000 Mathematics Subject Classification. Primary 17A40; Secondary 17B60, 17 B99.

Key words and phrases. Kantor pairs, Lie triple systems, Lie algebras.

The first author was partially supported by the MEC and Fondos FEDER, MTM2004-06580C02-01 and MTM2007-62390, and by the Plan de Investigación del Principado de Asturias FICYTIB05-017.

The second author was partially supported by the MEC and Fondos FEDER, MTM2004-03845 and MTM2007-61978, by the Plan de Investigación del Principado de Asturias FICYT-IB05-017, and by the Junta de Andalucía FQM264.

The third author was partially supported by Natural Sciences and Engineering Research Council of Canada Discovery Grant \#8836-2006. 
the corresponding property of $\mathcal{L}_{V}$. We will prove that this is indeed the case $(2.5$, $2.6,2.7)$.

One can go from the Kantor pair $V$ to its standard Lie envelope $\mathcal{L}_{V}$ in two steps, first from $V$ to the associated polarized Lie triple system $T(V)$ and then from the Lie triple system to its standard Lie envelope $\mathcal{L}_{T(V)}$ :

$$
\text { Kantor pair « Lie triple system « Lie algebra }
$$

The transfer of properties between $V$ and $T(V)$ is straightforward (Prop. 1.9). The heart of the matter lies in the relations between the Lie triple system $T(V)$ and its standard Lie envelope $\mathcal{L}_{T(V)}$. It turns out that our methods of dealing with this transfer are general enough to deal with Lie triple systems per se, and not only those that arise from Kantor pairs. In particular, we show (2.4) that a Lie triple system $T$ is nondegenerate if and only of its standard embedding $\mathcal{L}_{T}$ is so. We can even go one step further, and simply consider $\mathbb{Z}_{2}$-graded Lie algebras. The connection to Lie triple systems is given by the fact that the standard Lie envelope of a Lie triple system is a $\mathbb{Z}_{2}$-graded Lie algebra. We show in 2.3 that a $\mathbb{Z}_{2}$-graded Lie algebra is graded-nondegenerate if and only if it is nondegenerate.

One of the important questions in any structure theory is that of nondegeneracy of a simple object. Since there are prime degenerate Jordan pairs, there are of course prime degenerate Kantor pairs. However, we are able to prove (3.1) that this cannot happen for simple Kantor pairs as considered in this paper, namely over a ring $\Phi$ of scalars containing $1 / 2,1 / 3,1 / 5$. The basis for this is a result of Zelmanov [17], which says that the subalgebra generated by all absolute zero divisors of a Lie algebra $L$ over a ring of scalars with $1 / 6$ is locally nilpotent. This can be applied in our study of Kantor pairs, since it is easy to show that the subalgebra generated by all absolute zero divisors of a simple short $\mathbb{Z}$-graded Lie algebra $L=L_{-2} \oplus L_{-1} \oplus L_{0} \oplus L_{1} \oplus L_{2}$ is in fact an ideal.

We also consider the corresponding question for Lie triple systems: Is a simple Lie triple system $T$ nondegenerate? Our answer is yes, if $T$ is defined over a field of characteristic 0 (3.1). Our proof uses a result of Grishkov [9] which implies that simple Lie algebras in characteristic zero are always nondegenerate, i.e., do not have nonzero absolute zero divisors.

The final section of our paper contains some consequences of these results. We obtain a nice characterization of annihilators of ideals in nondegenerate Lie triple systems (3.2) and Kantor pairs (3.3), which easily implies the inheritance of nondegeneracy by ideals of Lie triple systems and Kantor pairs (3.4). We also study when Kantor pairs are Jordan pairs, and we do so by defining the biggest ideal of a Kantor pair which is a Jordan pair. For nondegenerate Kantor pairs, this ideal is essential if and only if the Kantor pair is in fact a Jordan pair (3.7).

\section{Preliminaries on Lie triple systems and Kantor Pairs}

Throughout this paper we will be dealing with Lie triple systems, Kantor pairs and Lie algebras over a ring of scalars $\Phi$ with $1 / 6 \in \Phi$.

1.1. We first recall some concepts for Lie algebras. An absolute zero divisor in a Lie algebra $L$ is an element $x \in L$ satisfying $[x,[x, L]]=0$. A Lie algebra is called nondegenerate if 0 is its only absolute zero divisor. We note that any ideal of a nondegenerate Lie algebra is again nondegenerate, see [18, Lemma 4]. The 
annihilator of an ideal $I$ in a Lie algebra $L$ is defined as $\operatorname{Ann}_{L}(I)=\{x \in L \mid$ $[x, I]=0\}$. If $L$ is nondegenerate, one knows $[5,2.5])$ that

$$
\operatorname{Ann}_{L}(I)=\{x \in L \mid[x,[I, x]]=0\} .
$$

A Lie algebra $L$ is called prime (respectively semiprime) if for all ideals $I, J$ of $L$ we have $[I, J]=0 \Rightarrow I=0$ or $J=0$ (resp. $[I, I]=0 \Rightarrow I=0$ ), and it is called strongly prime if $L$ is prime and nondegenerate.

1.2. A Lie triple system $T$ is a $\Phi$-module with a trilinear product $T \times T \times T \rightarrow T$ satisfying

$$
\begin{aligned}
0 & =[x, x, y] \\
0 & =[x, y, z]+[y, z, x]+[z, x, y], \\
{[x, y,[z, w, u]] } & =[[x, y, z], w, u]+[z,[x, y, w], u]+[z, w,[x, y, u]] .
\end{aligned}
$$

A map $d: T \rightarrow T$ is a derivation of $T$ if $d[x, y, z]=[d x, y, z]+[x, d y, z]+[x, y, d z]$ for every $x, y, z \in T$. We denote by $\operatorname{Der}(T)$ the set of all derivations of $T$, which is a Lie subalgebra of $\operatorname{End}(T)^{(-)}$. For every $x, y \in T$, the map $L(x, y): T \rightarrow T$ defined by $L(x, y)(z):=[x, y, z]$ is an (inner) derivation of $T$. The subalgebra generated by all inner derivations of $T$ is denoted by $L(T, T)$.

Every Lie algebra $L$ can be viewed as a Lie triple system if we define the product by: $[x, y, z]:=[[x, y], z]$ for every $x, y, z \in L$. Thus every submodule $T$ of $L$ such that $[[T, T], T] \subset T$ is an example of Lie triple system. In particular, if $L$ is a $\mathbb{Z}_{2}$-graded Lie algebra, $L=L_{0} \oplus L_{1}$, then $L_{1}$ with product $[x, y, z]:=[[x, y], z]$ is a Lie triple system. Note that under our assumptions on $\Phi$, a $\mathbb{Z}_{2}$-grading is the same as the eigenspace decomposition of an automorphism of $L$ of period 2 . In fact, $T$ is a Lie triple system if and only if there exists a $\mathbb{Z}_{2}$-graded Lie algebra $L=L_{0} \oplus L_{1}$ with $T=L_{1}$. Indeed, if $T$ is a Lie triple system and $D$ is a Lie algebra of derivations of $T$ such that $L(T, T) \subset D$, then $D \oplus T$ with product $\left[d_{1} \oplus x_{1}, d_{2} \oplus x_{2}\right]=\left[d_{1}, d_{2}\right]+L\left(x_{1}, x_{2}\right) \oplus\left(d_{1} x_{2}-d_{2} x_{1}\right)$ is a Lie algebra. In particular, $\mathcal{L}_{T}=L(T, T) \oplus T$ is a $\mathbb{Z}_{2}$-graded Lie algebra which is called the standard embedding of $T$, see for example [10, p. 309] or [15, IV]. The automorphism $\theta$ of $\mathcal{L}_{T}$ defining the $\mathbb{Z}_{2}$-grading of $\mathcal{L}_{T}$ is also called the main involution of $\mathcal{L}_{T}$.

1.3. An ideal $I$ of a Lie triple system $T$ is a $\Phi$-submodule of $T$ satisfying $[I, T, T] \subset$ $T$ (note that then $[T, I, T]+[T, T, I] \subset I$ by $(\operatorname{LTS} 1)$ and $(\operatorname{LTS} 2)$ ). A Lie triple system $T$ is simple if it has nontrivial product and contains no nontrivial ideals. An element in a Lie triple system $T$ is an absolute zero divisor if $[x, T, x]=0$. A Lie triple system without nonzero absolute zero divisors is called nondegenerate. We will say that a Lie triple system $T$ is semiprime if $[I, T, I] \neq 0$ for every nonzero ideal $I$ of $T$, and that $T$ is prime if every two nonzero ideals $I, J$ of $T$ give $[I, T, J] \neq 0$. Note that a Lie triple system is prime if and only if it is semiprime and every pair of nonzero ideals of $T$ have nonzero intersection. If $I$ is an ideal of a Lie triple system $T$ we define the annihilator of $I$ in $T$ as $\operatorname{Ann}_{T}(I)=\{x \in T \mid[x, I, T]=[T, I, x]=$ $0\}=T \cap \operatorname{Ann}_{\mathcal{L}_{T}}([T, I] \oplus I)$ where $[T, I] \oplus I$ is the ideal of $\mathcal{L}_{T}$ generated by $T$. It follows that $\operatorname{Ann}_{T}(I)$ is an ideal of $T$. Analogous to the case of Lie algebras, $T$ is semiprime if and only if $I \cap \operatorname{Ann}_{T}(I)=0$ for every ideal $I$ of $T$.

1.4. Concerning the transfer of properties between $T$ and its standard embedding $\mathcal{L}_{T}, T$ is (semi)prime if and only if $\left(\mathcal{L}_{T}, \theta\right)$ is (semi)prime as algebra with involution, see $[4,3.1]$. It also known [13, Th. 2.13], [15, VI, Th. 2 and Th. 3] that a Lie triple 
system is simple if and only if its standard embedding is either simple or a direct sum of two simple Lie algebras. In particular, this implies that the standard embedding of a prime Lie triple system need not be simple.

1.5. Recall that a Kantor pair $V=\left(V^{+}, V^{-}\right)$consists of two $\Phi$-modules with trilinear products $\{,\}:, V^{\sigma} \times V^{-\sigma} \times V^{\sigma} \rightarrow V^{\sigma}, \sigma= \pm$, that satisfy

$$
\begin{aligned}
{\left[V_{x, y}, V_{z, w}\right] } & =V_{\{x, y, z\}, w}-V_{z,\{y, x, w\}} \\
K_{K_{z, w} x, y} & =K_{z, w} V_{x, y}+V_{y, x} K_{z, w}
\end{aligned}
$$

where $[A, B]:=A B-B A, V_{x, y} z:=\{x, y, z\}$, and $K_{a, b} z:=\{a, z, b\}-\{b, z, a\}[1$, p.533]. Note that the opposite $V^{\mathrm{op}}:=\left(V^{-}, V^{+}\right)$of a Kantor pair is again a Kantor pair. Also, a Jordan pair is a Kantor pair with $K_{a, b}=0$ [14, 2.2]. Thus, Kantor pairs are generalizations of Jordan pairs.

If $L$ is a $\mathbb{Z}$-graded Lie algebra of the form $L=L_{-2} \oplus L_{-1} \oplus L_{0} \oplus L_{1} \oplus L_{2}$, then the pair of $\Phi$-modules $\left(L_{1}, L_{-1}\right)$ with product $\{x, y, z\}:=[[x, y], z]$, for every $x, z \in L_{\sigma}$, $y \in L_{-\sigma}$, is a Kantor pair. Conversely, it follows from [1] or [3, Th. 4.3 and Cor. 4.6] that for any Kantor pair $V=\left(V^{+}, V^{-}\right)$there exists a unique up to isomorphism 5-graded Lie algebra $L=L_{-2} \oplus L_{-1} \oplus L_{0} \oplus L_{1} \oplus L_{2}$ with the following properties: (i) $V$ isomorphic to the Kantor pair $\left(L_{1}, L_{-1}\right)$, (ii) $L_{2 \sigma}=\left[L_{\sigma}, L_{\sigma}\right], L_{0}=\left[L_{\sigma}, L_{-\sigma}\right]$ for $\sigma= \pm$, and (iii) if $\left[x_{-2}+x_{0}+x_{2}, L_{1} \oplus L_{-1}\right]=0$ then $x_{-2}+x_{0}+x_{2}=0$. Thus, after identifying $V \equiv\left(L_{1}, L_{-1}\right)$ the products in $L$ are $[[x, z], y]=K_{x, z} y$, $[[x, y], z]=V_{x, y} z$ and $[[y, x], z]=-V_{x, y} z$ for $x, z \in L_{\sigma}, y \in L_{-\sigma}$. We will call this Lie algebra the standard embedding of $V$ and denote it $\mathcal{L}_{V}$. In the setting of what are now called Kantor triple systems, the construction of $\mathcal{L}_{V}$ goes back to the fundamental papers [11] and [12] of Kantor. Notice also that $V$ is a Jordan pair if and only if $L_{2}$ and $L_{-2}$ in the above construction are both zero. In this case, $\mathcal{L}_{V}$ is the Tits-Kantor-Koecher algebra of $V$.

1.6. Let $V$ be a Kantor pair. A pair $I=\left(I^{+}, I^{-}\right)$of $\Phi$-submodules of $V$ is an ideal of $V$ if $\left\{I^{\sigma}, V^{-\sigma}, V^{\sigma}\right\}+\left\{V^{\sigma}, I^{-\sigma}, V^{\sigma}\right\}+\left\{V^{\sigma}, V^{-\sigma}, I^{\sigma}\right\} \subset I^{\sigma}$ for $\sigma= \pm$. A Kantor pair $V$ is simple if it has nontrivial product and $V$ contains no nontrivial ideals. An element $x \in V^{\sigma}$ is an absolute zero divisor of $V$ if $\left\{x, V^{-\sigma}, x\right\}=0$. A Kantor pair without nonzero absolute zero divisors is called nondegenerate. A Kantor pair $V$ is semiprime if every nonzero ideal $I=\left(I^{+}, I^{-}\right)$of $V$ has $\left\{I^{+}, V^{-}, I^{+}\right\} \neq 0$ or $\left\{I^{-}, V^{+}, I^{-}\right\} \neq 0$. We will say that a Kantor pair is prime if for any two ideals $I$ and $J$ of $V,\left\{I^{\sigma}, V^{-\sigma}, J^{\sigma}\right\}=0, \sigma= \pm$, implies $I=0$ or $J=0$. Note that a Kantor pair is prime if and only if it is semiprime and every two nonzero ideals of $V$ have nonzero intersection.

Let $V$ be a Kantor pair and $I=\left(I^{+}, I^{-}\right)$and ideal of $V$. We define the annihilator of $I$ in $V$ as $\left(\operatorname{Ann}_{V}(I)^{+}, \operatorname{Ann}_{V}(I)^{-}\right)$with

$$
\begin{aligned}
\operatorname{Ann}_{V}(I)^{\sigma}=\left\{x \in V^{\sigma} \mid\right. & \left\{V^{\sigma}, I^{-\sigma}, x\right\}=\left\{I^{\sigma}, V^{-\sigma}, x\right\}=\left\{x, I^{-\sigma}, V^{\sigma}\right\} \\
& \left.=\left\{V^{-\sigma}, x, I^{-\sigma}\right\}=\left\{I^{-\sigma}, x, V^{-\sigma}\right\}=\left\{x, V^{-\sigma}, I^{\sigma}\right\}=0\right\}
\end{aligned}
$$

As for Lie triple systems, we have that $\operatorname{Ann}_{V}(I)=\left(\operatorname{Ann}_{V}(I)^{+}, \operatorname{Ann}_{V}(I)^{-}\right)$is an ideal of $V$. If $\mathcal{I}$ is the ideal of $\mathcal{L}_{V}$ generated by $I$, then $\operatorname{Ann}_{V}(I)=V \cap \operatorname{Ann}_{\mathcal{L}_{V}}(\mathcal{I})$. 
1.7. By $[1$, Th. 7$]$ each Kantor pair $V$ gives rise to a Lie triple system $T(V)=$ $V^{+} \oplus V^{-}$whose product is given by

$$
\begin{aligned}
& {\left[x^{+}+x^{-}, y^{+}+y^{-}, z^{+}+z^{-}\right]=} \\
& \quad=\left(\left\{x^{+}, y^{-}, z^{+}\right\}+\left\{x^{+}, z^{-}, y^{+}\right\}-\left\{y^{+}, z^{-}, x^{+}\right\}-\left\{y^{+}, x^{-}, z^{+}\right\}\right) \oplus \\
& \quad \oplus\left(\left\{x^{-}, y^{+}, z^{-}\right\}+\left\{x^{-}, z^{+}, y^{-}\right\}-\left\{y^{-}, z^{+}, x^{-}\right\}-\left\{y^{-}, x^{+}, z^{-}\right\}\right)
\end{aligned}
$$

for $x^{+}+x^{-}, y^{+}+y^{-}, z^{+}+z^{-} \in T(V)$, called the associated Lie triple system. Moreover, if $V$ is a Kantor pair and $T(V)$ its associated Lie triple system, their standard envelopes $\mathcal{L}_{V}$ and $\mathcal{L}_{T(V)}$ are isomorphic as Lie algebras: if $\mathcal{L}_{V}=L_{-2} \oplus$ $L_{-1} \oplus L_{0} \oplus L_{1} \oplus L_{2}$ is the standard envelope of $V$ given in (1.5), then $\mathcal{L}_{T(V)}=$ $\left(\mathcal{L}_{T(V)}\right)_{0} \oplus\left(\mathcal{L}_{T(V)}\right)_{1}$ with $\left(\mathcal{L}_{T(V)}\right)_{0}=L_{-2} \oplus L_{0} \oplus L_{2}$ and $\left(\mathcal{L}_{T(V)}\right)_{1}=L_{-1} \oplus L_{1}$.

There are close relations between ideals of a Kantor pair $V$ and ideals of $T(V)$ :

Lemma 1.8. Let $V$ be a Kantor pair and $T(V)$ its associated Lie triple system.

(1) If $I=\left(I^{+}, I^{-}\right)$is an ideal $V$, then $I^{+} \oplus I^{-}$is an ideal of $T(V)$.

(2) If $\tilde{I}$ is an ideal of $T(V)$, then $\tilde{I} \cap V:=\left(\tilde{I} \cap V^{+}, \tilde{I} \cap V^{-}\right)$and $\pi(\tilde{I}):=$ $\left(\pi^{+}(\tilde{I}), \pi^{-}(\mathcal{I})\right)$ are ideals of $V$, where $\pi^{\sigma}: V^{+} \oplus V^{-} \rightarrow V^{\sigma}$ denotes the projection onto $V^{\sigma}$ along $V^{-\sigma}$.

(3) If $I=\left(I^{+}, I^{-}\right)$is an ideal $V, \operatorname{Ann}_{T(V)}\left(I^{+} \oplus I^{-}\right)=\operatorname{Ann}_{V}(I)^{+} \oplus \operatorname{Ann}_{V}(I)^{-}$.

Proof. (1) is trivial.

(2) That $\tilde{\pi}(I)$ is an ideal follows from the following product rules with obvious notations: $\pi^{\sigma}\left(\left[x, y^{-\sigma}, z^{\sigma}\right]\right)=\pi^{\sigma}\left(\left\{x^{\sigma}, y^{-\sigma}, z^{\sigma}\right\} \oplus\left\{x^{-\sigma}, z^{\sigma}, y^{-\sigma}\right\}-\left\{y^{-\sigma}, z^{\sigma}, x^{-\sigma}\right\}\right)=$ $\left\{\pi^{\sigma}(x), y^{-\sigma}, z^{\sigma}\right\}$ and, similarly, $\pi^{\sigma}\left(\left[x^{\sigma}, y, z^{\sigma}\right]\right)=\left\{x^{\sigma}, \pi^{-\sigma}(y), z^{\sigma}\right\}$ and $\pi^{\sigma}\left(\left[x^{\sigma}, y^{-\sigma}, z\right]\right)=$ $\left\{x^{\sigma}, y^{-\sigma}, \pi^{\sigma}(z)\right\}$.

(3) It is clear that $\operatorname{Ann}_{V}(I)^{+} \oplus \operatorname{Ann}_{V}(I)^{-} \subset \operatorname{Ann}_{T(V)}\left(I^{+} \oplus I^{-}\right)$. Moreover, by the formulas above, if $z \in \operatorname{Ann}_{T(V)}\left(I^{+} \oplus I^{-}\right)$, then $\pi^{\sigma}(z) \in \operatorname{Ann}_{T(V)}\left(I^{+} \oplus I^{-}\right)$. Now, $\operatorname{Ann}_{T(V)}\left(I^{+} \oplus I^{-}\right) \cap V^{\sigma} \subset \operatorname{Ann}_{V}(I)^{\sigma}$ implies (iii).

The last lemma allows us to relate regularity properties of a Kantor pair with those of its associated Lie triple system $T(V)$ :

Proposition 1.9. Let $V$ be a Kantor pair and $T(V)$ its associated Lie triple system. Then

(1) Every absolute zero divisor of $V$ is an absolute zero divisor of $T(V)$. Moreover, if $x^{+}+x^{-} \in T(V)$ is an absolute zero divisor of $T(V)$, then $x^{+}$and $x^{-}$are absolute zero divisors of $V$.

(2) $V$ is nondegenerate, semiprime, prime or simple if and only if $T(V)$ is so.

Proof. (1) On the one hand, if $\left[x^{+}+x^{-}, V^{+} \oplus V^{-}, x^{+}+x^{-}\right]=0$, then, for every $a \in V^{+}, 0=\left[x^{+}+x^{-}, a, x^{+}+x^{-}\right]=\left(\left\{x^{+}, x^{-}, a\right\}-2\left\{a, x^{-}, x^{+}\right\}\right) \oplus\left\{x^{-}, a, x^{-}\right\}$, whence $\left\{x^{-}, V^{+}, x^{-}\right\}=0$. Similarly, $\left\{x^{+}, V^{-}, x^{+}\right\}=0$. On the other hand, if $\left\{x^{\sigma}, V^{-\sigma}, x^{\sigma}\right\}=0$, then $\left[x^{\sigma}, V^{+} \oplus V^{-}, x^{\sigma}\right]=\left\{x^{\sigma}, V^{-\sigma}, x^{\sigma}\right\}=0$.

(2) That $V$ is nondegenerate if and only if $T(V)$ is so, follows from (1). To show that $V$ (semi)prime implies $T(V)$ (semi)prime, let $\tilde{I}, \tilde{J}$ be two nonzero ideals of $T(V)$ ( $I=J$ for semiprimeness) and suppose that $[\tilde{I}, T(V), \tilde{J}]=0$. Then $\pi(\tilde{I}), \pi(\tilde{J})$ are nonzero ideals of $V$ such that $\left\{\pi^{\sigma}(\tilde{I}), V^{-\sigma}, \pi^{\sigma}(\tilde{J})\right\} \subset \pi^{\sigma}\left[\tilde{I}, V^{-\sigma}, \tilde{J}\right]=0, \sigma= \pm$, contradiction. 
Conversely, suppose that $T(V)$ is semiprime but $V$ is not. We claim that there exists a nonzero ideal $I$ of $V$ such that

$$
\left\{I^{\sigma}, V^{-\sigma}, I^{\sigma}\right\}=\left\{I^{\sigma}, I^{-\sigma}, V^{\sigma}\right\}=\left\{V^{\sigma}, I^{-\sigma}, I^{\sigma}\right\}=0, \quad \sigma= \pm .
$$

Indeed, by assumption there exists a nonzero ideal $J$ of $V$ with $\left\{J^{\sigma}, V^{-\sigma}, J^{\sigma}\right\}=0$, $\sigma= \pm$. If $\left\{V^{\sigma}, J^{-\sigma}, V^{\sigma}\right\}=0$, then $J$ satisfies (a). Otherwise, the non-zero ideal $I=\left(\left\{V^{+}, J^{-}, V^{+}\right\},\left\{V^{-}, J^{+}, V^{-}\right\}\right) \subset J$ of $V$ satisfies (a), since for $y \in J^{\sigma}$, $a, b \in V^{-\sigma}$ and $x$ or $z$ belonging to $J^{\sigma}$ we have, by (KP1),

$$
\{x,\{a, y, b\}, z\}=-\{y, a,\{x, b, z\}\}+\{\{y, a, x\}, b, z\}+\{x, b,\{y, a, z\}\}=0,
$$

and so $\left\{J^{\sigma},\left\{V^{-\sigma}, J^{\sigma}, V^{-\sigma}\right\}, V^{\sigma}\right\}=0=\left\{V^{\sigma},\left\{V^{-\sigma}, J^{\sigma}, V^{-\sigma}\right\}, J^{\sigma}\right\}$. Let now $I$ be a nonzero ideal of $V$ satisfying (a). Then $I^{+} \oplus I^{-}$is a nonzero ideal of $T(V)$ such that $\left[I^{+} \oplus I^{-}, T(V), I^{+} \oplus I^{-}\right]=0$ because $\left[I^{\sigma}, V^{+} \oplus V^{-}, I^{\sigma}\right] \subset\left\{I^{\sigma}, V^{-\sigma}, I^{\sigma}\right\}=0$ and $\left[I^{\sigma}, V^{+} \oplus V^{-\sigma}, I^{-\sigma}\right] \subset\left\{I^{\sigma}, I^{-\sigma}, V^{\sigma}\right\}+\left\{V^{\sigma}, I^{-\sigma}, I^{\sigma}\right\}+\left\{V^{-\sigma}, I^{\sigma}, I^{-\sigma}\right\}=0$ for $\sigma= \pm$. Thus $T(V)$ semiprime implies $V$ semiprime. If $T(V)$ is prime, then $V$ is semiprime. Hence to show that in this case $V$ is actually prime, it suffices to prove that every two nonzero ideals of $V$ have nonzero intersection. But this is clear, since it holds in $T(V)$.

If $T(V)$ is simple, $V$ is simple by $(1.8)(1)$. Conversely, let us suppose that $V$ is simple and let $\tilde{I}$ be a nonzero ideal of $T(V)$. Then, by $(1.8)(2),\left(\pi^{+}(\tilde{I}), \pi^{-}(\tilde{I})\right)$ is a nonzero ideal of $V$, so it is equal to $V$. It now follows that $0 \neq\left\{V^{\sigma}, V^{-\sigma}, V^{\sigma}\right\}=$ $\left\{V^{\sigma}, \pi^{-\sigma}(\tilde{I}), V^{\sigma}\right\}=\left\{V^{\sigma}, \tilde{I}, V^{\sigma}\right\} \subset V^{\sigma} \cap \tilde{I}$. The ideal $\tilde{I} \cap V$ is therefore nonzero, hence equal to $V$ which implies $\tilde{I}=T(V)$.

\section{Relations With Standard Lie EnVElopes}

The main result of this paragraph is the transfer of nondegeneracy between Lie triple systems and their standard envelopes. As a corollary we then get the analogous result for Kantor pairs. For the sake of completeness, we also include results about the transfer of simplicity between Kantor pairs and Lie envelopes, which are due to Allison and Smirnov.

In the following lemma, capital letters denote adjoint maps of elements of $L$, i.e., $X=\operatorname{ad}_{x}, A=\operatorname{ad} a$, for $x, a \in L$.

Lemma 2.1. Let $L$ be a Lie algebra and let $x, y \in L$ absolute zero divisors of $L$. Then:

(1) $[x, y]$ and, for every $a \in L,[[x, a],[[x, a], a]]$ are absolute zero divisors of $L$.

(2) For every $a_{1}, a_{2}, a_{3} \in L$ and $\sigma \in S_{3}$ we have

$$
\left[\left[x, a_{1}\right],\left[\left[x, a_{2}\right], a_{3}\right]\right]=\left[\left[x, a_{\sigma(1)}\right],\left[\left[x, a_{\sigma(2)}\right], a_{\sigma(3)}\right]\right] .
$$

Proof. (1) That $[x, y]$ is an absolute zero divisor follows from $(X Y-Y X)^{2}=$ $X Y X Y-X Y Y X-Y X X Y+Y X Y X=0$. Let us consider $b=[[x, a],[[x, a], a]]$. Then $\operatorname{ad}_{b}^{2}=9 X A^{2} X A^{2} X A^{2} X A^{2} X=0$ by [7, Prop. 1.7(3)], so $b$ is an absolute zero divisor of $L$.

(2) First notice that for any $a_{1}, a_{2} \in L,\left[\left[x, a_{1}\right],\left[x, a_{2}\right]\right]=0$ because

$$
\left[\left[x, a_{1}\right],\left[x, a_{2}\right]\right]=\left[\left[\left[x, a_{1}\right], x\right], a_{2}\right]-\left[x,\left[a_{2},\left[x, a_{1}\right]\right]\right]=0 .
$$


Therefore $\left[\left[x, a_{1}\right],\left[\left[x, a_{2}\right], a_{3}\right]\right]=\left[\left[x, a_{2}\right],\left[\left[x, a_{1}\right], a_{3}\right]\right]$. Moreover,

$$
\begin{aligned}
{\left[\left[x, a_{1}\right],\left[\left[x, a_{2}\right], a_{3}\right]\right] } & =\left[\left[x, a_{1}\right],\left[\left[x, a_{3}\right], a_{2}\right]\right]+\left[\left[x, a_{1}\right],\left[x,\left[a_{2}, a_{3}\right]\right]\right] \\
& =\left[\left[x, a_{1}\right],\left[\left[x, a_{3}\right], a_{2}\right]\right] .
\end{aligned}
$$

The formulae above prove (2).

In the following we will use a generalization of the Jordan algebras of Lie algebras given in [6]. Let $L$ be a Lie subalgebra of a Lie algebra $L^{\prime}$. We will say that an element $x \in L^{\prime}$ is a $L$-Jordan element if $\operatorname{ad}_{x}^{3} L=0$ and $[[L, x], L] \subset L$.

Theorem 2.2. Let $L$ be a Lie subalgebra of a Lie algebra $L^{\prime}$ and $x \in L^{\prime}$ a $L$ Jordan element. Then $L$ with the new product defined by $a \bullet b:=\frac{1}{2}[[a, x], b]$ is a nonassociative algebra, denoted by $L^{(x)}$, such that:

(1) $\operatorname{ker}_{L}(x):=\{a \in L \mid[x,[x, a]]=0\}$ is an ideal of $L^{(x)}$.

(2) $L_{x}:=L^{(x)} / \operatorname{ker}_{L}(x)$ is a Jordan algebra, with $U$-operator given by $U_{\bar{a}} \bar{b}=$ $\frac{1}{8} \overline{\operatorname{ad}_{a}^{2} \operatorname{ad}_{x}^{2} b}$, for all $a, b \in I$, where $\bar{a}$ denotes the coset of a with respect to $\operatorname{ker}_{I}(x)$. This Jordan algebra is called the generalized Jordan algebra of $L$ at $x$.

(3) If $L$ is nondegenerate or strongly prime, so is $L_{x}$.

Proof. The proofs of (1) and (2) are identical to [6, 2.4 (1)(2)], taking into account that $[6,2.3]$ can be rewritten under the conditions of this theorem and that the identities given there are true over $L ;(3)$ follows in the same way as $[6,2.15(1)]$ and $[8,2.2]$.

We say that a $\mathbb{Z}_{2}$-graded Lie algebra $L=L_{0} \oplus L_{1}$ is graded-nondegenerate if it has no absolute zero divisors in $L_{0} \cup L_{1}$. In the following result we will show that the notions of nondegeneracy and graded-nondegeneracy are equivalent.

Theorem 2.3. Let $L=L_{0} \oplus L_{1}$ be a $\mathbb{Z}_{2}$-graded Lie algebra. Then $L$ is gradednondegenerate if and only if it is nondegenerate.

Proof. It is of course sufficient to show that a graded-nondegenerate $\mathbb{Z}_{2}$-graded Lie algebra $L$ cannot have nonzero absolute zero divisors. Suppose that $x_{0}+x_{1}$ is an absolute zero divisor of $L$. Then, for $i=0,1,0=\left[x_{0}+x_{1},\left[x_{0}+x_{1}, L_{i}\right]\right]=$ $\left[x_{0},\left[x_{0}, L_{i}\right]\right]+\left[x_{0},\left[x_{1}, L_{i}\right]\right]+\left[x_{1},\left[x_{0}, L_{i}\right]\right]+\left[x_{1},\left[x_{1}, L_{i}\right]\right]$. Considering homogeneous components,

$$
\begin{array}{r}
\operatorname{ad}_{x_{0}}^{2}+\operatorname{ad}_{x_{1}}^{2}=0 \\
\operatorname{ad}_{x_{0}} \operatorname{ad}_{x_{1}}+\operatorname{ad}_{x_{1}} \operatorname{ad}_{x_{0}}=0 .
\end{array}
$$

Therefore $\left[x_{0}-x_{1},\left[x_{0}-x_{1}, L\right]\right]=\left[x_{0},\left[x_{0}, L\right]\right]-\left[x_{0},\left[x_{1}, L\right]\right]-\left[x_{1},\left[x_{0}, L\right]\right]+\left[x_{1},\left[x_{1}, L\right]\right]=$ 0 and $x_{0}-x_{1}$ is an absolute zero divisor of $L$. Now, $\left[x_{0}+x_{1}, x_{0}-x_{1}\right]=-\left[x_{0}, x_{1}\right]+$ $\left[x_{1}, x_{0}\right]=2\left[x_{1}, x_{0}\right] \in L_{1}$, which implies $\left[x_{1}, x_{0}\right]=0$, because, by $(2.1)(1),\left[x_{0}+\right.$ $\left.x_{1}, x_{0}-x_{1}\right]$ is an absolute zero divisor. Then $\operatorname{ad}_{x_{0}} \operatorname{ad}_{x_{1}}=\operatorname{ad}_{x_{1}} \operatorname{ad}_{x_{0}}$, and by (b)

$$
\operatorname{ad}_{x_{0}} \operatorname{ad}_{x_{1}}=\operatorname{ad}_{x_{1}} \operatorname{ad}_{x_{0}}=0 .
$$

Finally, we have $\operatorname{ad}_{x_{1}}^{3}=-\operatorname{ad}_{x_{0}}^{2} \operatorname{ad}_{x_{1}}=0$ and for any $z, t \in L$, using (a) and (c),

$$
\begin{aligned}
-\left[\left[x_{1},\left[x_{1}, z\right]\right],\left[x_{1}, t\right]\right] & =\left[\left[x_{0},\left[x_{0}, z\right]\right],\left[x_{1}, t\right]\right] \\
& =\left[\left[\left[x_{0},\left[x_{0}, z\right]\right], x_{1}\right], t\right]+\left[x_{1},\left[\left[x_{0},\left[x_{0}, z\right]\right], t\right]\right] \\
& =\left[x_{1},\left[\left[x_{0}, t\right],\left[x_{0}, z\right]\right]\right]+\left[x_{1},\left[x_{0},\left[\left[x_{0}, z\right], t\right]\right]\right]=0 .
\end{aligned}
$$


So, for $y=\left[x_{1},\left[x_{1}, z\right]\right]$ we get $\left[y,\left[x_{1}, t\right]\right]=0$ and then

$$
\begin{aligned}
{[y,[y, t]] } & =\left[y,\left[\left[x_{1},\left[x_{1}, z\right]\right], t\right]\right] \\
& =\left[y,\left[\left[x_{1}, t\right],\left[x_{1}, z\right]\right]\right]+\left[y,\left[x_{1},\left[\left[x_{1}, z\right], t\right]\right]\right]=0
\end{aligned}
$$

since $\left[y,\left[\left[x_{1}, t\right],\left[x_{1} z\right]\right]\right]=\left[\left(\left[y,\left[x_{1}, t\right]\right]\right),\left[x_{1}, t\right]\right]+\left[\left[x_{1}, t\right],\left(\left[y,\left[x_{1}, z\right]\right]\right)\right]=0$. If we take any $z \in L_{i}, i=0,1$, then $y=\left[x_{1},\left[x_{1}, z\right]\right] \in L_{i}$ is an absolute zero divisor of $L$ by (d). By hypothesis, $\left[x_{1},\left[x_{1}, z\right]\right]=0$ for any $z \in L_{0} \cup L_{1}$, so $\left[x_{1},\left[x_{1}, L\right]\right]=0$, which implies that $x_{1}=0$ because $L$ has no absolute zero divisors in $L_{1}$. Then $x_{0}+x_{1}=x_{0} \in L_{0}$ is an absolute zero divisor of $L$, so it must be zero.

Now we show that nondegeneracy is an equivalent condition for a Lie triple system and its standard envelope.

Theorem 2.4. Let $T$ be a Lie triple system and $\mathcal{L}_{T}=L_{0} \oplus L_{1}$ be its standard envelope. Then $\mathcal{L}_{T}$ is nondegenerate if and only if $T$ is nondegenerate.

Proof. Let us suppose that $\mathcal{L}_{T}$ is nondegenerate and that there exists $x \in L_{1}$ with $\left[x,\left[x, L_{1}\right]\right]=0$. Then, $\operatorname{ad}_{x}^{3}\left(\mathcal{L}_{T}\right)=\operatorname{ad}_{x}^{3}\left(L_{0}\right) \subset \operatorname{ad}_{x}^{2}\left(L_{1}\right)=0$. So $x$ is ad-nilpotent in $\mathcal{L}_{T}$ of index $\leq 3$ and we can consider the Jordan algebra $\left(\mathcal{L}_{T}\right)_{x}$ of $\mathcal{L}_{T}$ at $x$ as in (2.2). By hypothesis, $\left[x,\left[x, L_{1}\right]\right]=0$ so we can assume that all elements of $\left(\mathcal{L}_{T}\right)_{x}$ are of the form $a+\operatorname{ker}_{\mathcal{L}_{T}} x$, with $a \in L_{0}$. Now,

$$
\left[x,\left[x,\left[\left[L_{0}, x\right], L_{0}\right]\right]\right] \subset\left[x,\left[x, L_{1}\right]\right]=0
$$

which implies that the Jordan algebra $\left(\mathcal{L}_{T}\right)_{x}$ has trivial (Jordan) product and, since $\left(\mathcal{L}_{T}\right)_{x}$ is nondegenerate by $(2.2)(3),\left(\mathcal{L}_{T}\right)_{x}=0$, i.e., $\left[x,\left[x, \mathcal{L}_{T}\right]\right]=0$. Hence $x=0$ because $\mathcal{L}_{T}$ is nondegenerate.

Conversely, suppose that $T$ is nondegenerate and that $x \in L_{0} \cup L_{1}$ is an absolute zero divisor of $\mathcal{L}_{T}$. By nondegeneracy of $T$, we can suppose that $x \in L_{0}$. For any $y, y^{\prime} \in L_{1}$ and any $\lambda \in \Phi$, we have by (2.1)(1) that

$$
\left[\left[x, y+\lambda y^{\prime}\right],\left[\left[x, y+\lambda y^{\prime}\right], y+\lambda y^{\prime}\right]\right]
$$

is an absolute zero divisor of $\mathcal{L}_{T}$ contained in $L_{1}$. It is therefore zero since $T$ is nondegenerate. Linearizing and using (2.1)(2)

$$
\begin{aligned}
0= & {\left[\left[\left[x, y+\lambda y^{\prime}\right],\left[\left[x, y+\lambda y^{\prime}\right], y+\lambda y^{\prime}\right]\right]=[[x, y],[[x, y], y]]+3 \lambda\left[[x, y],\left[[x, y], y^{\prime}\right]\right]\right.} \\
& +3 \lambda^{2}\left[[x, y],\left[\left[x, y^{\prime}\right], y^{\prime}\right]\right]+\lambda^{3}\left[\left[x, y^{\prime}\right],\left[\left[x, y^{\prime}\right], y^{\prime}\right]\right] .
\end{aligned}
$$

Evaluating this for $\lambda=1,2,3 \in \Phi$, shows $0=\left[[x, y],\left[[x, y], y^{\prime}\right]\right]$ for any $y^{\prime} \in L_{1}$, i.e., $[x, y] \in T$ is an absolute zero divisor, whence $[x, y]=0$. Now $x=0$ follows because the map $L_{0} \rightarrow$ ad $L_{0} \mid L_{1}$ is injective. Thus $\mathcal{L}_{T}$ is graded-nondegenerate, which by 2.3 is equivalent to being nondegenerate.

Since the standard envelope of a Kantor pair $V$ is isomorphic as a Lie algebra to the standard envelope of $T(V)$ and since by (1.9)(2) $V$ is nondegenerate if and only if $T(V)$ is nondegenerate, we get:

Corollary 2.5. A Kantor pair $V$ is nondegenerate if and only if its standard envelope $\mathcal{L}_{V}$ is a nondegenerate Lie algebra.

Proposition 2.6. (Allison-Smirnov [2]) Let $V$ be a Kantor pair and let $\mathcal{L}_{V}=$ $L_{2} \oplus L_{1} \oplus L_{0} \oplus L_{-1} \oplus L_{-2}$ be its standard envelope.

(1) For any ideal $\left(I^{+}, I^{-}\right)$of $V$, the ideal of $\mathcal{L}_{V}$ generated by $I^{+} \cup I^{-}$is $\operatorname{id}_{\mathcal{L}_{V}}\left(I^{+} \cup I^{-}\right)=\left[I^{+}, L_{1}\right] \oplus I^{+} \oplus\left(\left[I^{+}, L_{-1}\right]+\left[L_{1}, I^{-}\right]\right) \oplus I^{-} \oplus\left[I^{-}, L_{-1}\right]$. 
(2) $L_{2} \oplus L_{0} \oplus L_{-2}$ does not contain nonzero ideals of $\mathcal{L}_{V}$.

(3) If $\mathcal{L}_{V}$ is graded-semiprime, every nonzero ideal $\mathcal{I}$ of $\mathcal{L}_{V}$ contains a nonzero graded ideal. In particular, $\mathcal{I}$ has nonzero intersection with $L_{1} \oplus L_{-1}$.

(4) $\mathcal{L}_{V}$ is (semi)prime if and only if $\mathcal{L}_{V}$ is graded-(semi)prime.

Proof. (1) and (2) can easily be checked by the reader.

(3) Let $0 \neq x \in \mathcal{I} \triangleleft \mathcal{L}_{V}$ and write $x=x_{2}+x_{1}+x_{0}+x_{-1}+x_{-2}$ with $x_{i} \in L_{i}$. Let $i_{0}$ be the maximal index such that $x_{i_{0}} \neq 0$. The Lie algebra $\overline{\mathcal{L}}_{V}=\mathcal{L}_{V} / \mathcal{I}$ is pregraded in the sense of [16], i.e., denoting by $\bar{L}_{i} \subset \mathcal{L}_{V}$ the canonical images of $L_{i}$ we have $\overline{\mathcal{L}}_{V}=\sum_{i=-2}^{2} \bar{L}_{i}$ and $\left[\bar{L}_{i}, \bar{L}_{j}\right] \subset \bar{L}_{i+j}$. Note that $\bar{x}_{i_{0}} \in B\left(\overline{\mathcal{L}}_{V}\right)=$ $\sum_{k=-2}^{2}\left(\bar{L}_{k} \cap \sum_{i<k} \bar{L}_{i}\right)$. By [16, 2.1], $B\left(\overline{\mathcal{L}}_{V}\right)$ is a nilpotent ideal. In particular, $\operatorname{id}_{\overline{\mathcal{L}}_{V}}\left(\bar{x}_{i_{0}}\right)$ is nilpotent in $\overline{\mathcal{L}}_{V}$, i.e., there exists $m \in \mathbb{N}$ such that $\left(\operatorname{id}_{\mathcal{L}_{V}}\left(x_{i_{0}}\right)\right)^{(m)} \subset \mathcal{I}$. Moreover, since $\mathcal{L}_{V}$ is graded-semiprime and $\operatorname{id}_{\mathcal{L}_{V}}\left(x_{i_{0}}\right)$ is graded, $\left(\operatorname{id}_{\mathcal{L}_{V}}\left(x_{i_{0}}\right)\right)^{(m)}$ is a nonzero graded ideal contained in $\mathcal{I}$. That $\mathcal{I}$ has nonzero intersection with $L_{1} \oplus L_{-1}$ now follows from (1). (4) is immediate from (3).

Proposition 2.7. Let $V$ be a Kantor pair and let $\mathcal{L}_{V}$ be its standard envelope.

(1) If $V$ is semiprime, then $\mathcal{L}_{V}$ is semiprime.

(2) $V$ is prime if and only if $\mathcal{L}_{V}$ is prime and $V$ is semiprime.

(3) (Allison-Smirnov [2]) $\mathcal{L}_{V}$ is a simple Lie algebra if and only if $V$ is a simple Kantor pair.

Proof. (1) and (2). Since by (2.6)(2) graded ideals of $\mathcal{L}_{V}$ have a nonzero intersection with $V$, it follows that $V$ (semi)prime implies $\mathcal{L}_{V}$ graded-(semi)prime, hence (semi)prime by $(2.6)(3)$.

Conversely, if $V$ is semiprime, $\mathcal{L}_{V}$ is prime and $I=\left(I^{+}, I^{-}\right)$and $J=\left(J^{+}, J^{-}\right)$ are nonzero ideals of $V$, it suffices to show that $I \cap J \neq 0$. Let $\mathcal{K}:=\left[\operatorname{id}_{\mathcal{L}_{V}}(I), \operatorname{id}_{\mathcal{L}_{V}}(J)\right]$ which is a nonzero graded ideal of $\mathcal{L}_{V}$. So it has nonzero intersection with $L_{1} \oplus L_{-1}$. One can check that $\mathcal{K} \cap L_{1}=\left[\left[I^{+}, L_{1}\right], J^{-}\right]+\left[I^{+},\left[J^{+}, L_{-1}\right]\right]+\left[I^{+},\left[J^{-}, L_{1}\right]\right]+$ $\left[\left[I^{+}, L_{-1}\right], J^{+}\right]+\left[\left[I^{-}, L_{1}\right], J^{+}\right]+\left[\left[J^{+}, L_{1}\right], I^{-}\right] \subset I^{+} \cap J^{+}$, and similarly $\mathcal{K} \cap L_{-1} \subset$ $I^{-} \cap J^{-}$.

(3) If $\mathcal{L}_{V}$ is simple and $\left(I^{+}, I^{-}\right)$is a nonzero ideal of $V$, then $\operatorname{id}_{\mathcal{L}_{V}}\left(I^{+} \cup I^{-}\right)=\mathcal{L}_{V}$, so by $(2.6)(1)\left(I^{+}, I^{-}\right)=V$. Conversely, let $\mathcal{I}$ be a nonzero ideal of $\mathcal{L}_{V}$. If $V$ is simple, then $V$ is semiprime, so by (1) and (2.6)(3) $0 \neq\left(\mathcal{I} \cap L_{1}, \mathcal{I} \cap L_{-1}\right) \triangleleft V$. Simplicity of $V$ implies $\left(\mathcal{I} \cap L_{1}, \mathcal{I} \cap L_{-1}\right)=V$. Since $V$ generates $\mathcal{L}_{V}$ as algebra, $\mathcal{I}=\mathcal{L}_{V}$.

\section{Some Consequences}

Theorem 3.1. (1) A simple Kantor pair over a ring of scalars $\Phi$ containing $1 / 2,1 / 3,1 / 5$ is nondegenerate.

(2) A simple Lie triple system over a field of characteristic zero is nondegenerate.

Proof. (1) The standard enveloping algebra $L=\mathcal{L}_{V}$ is simple by (2.7)(3). The span $K_{1}(L)$ of all absolute zero divisors of $L$ is invariant under all automorphisms, hence in particular under all inner automorphism exp ad $x, x \in L_{i}, i \in\{ \pm 1, \pm 2\}$ (note that under our assumptions on $\Phi$ these maps are indeed automorphisms). It follows that $K_{1}(L)$ is an ideal. Since it is locally nilpotent by [17], it must be zero, i.e., $\mathcal{L}_{V}$ is nondegenerate, whence $V$ is nondegenerate by $(2.5)$. 
(2) By (2.5) it suffices to show that the standard embedding $L$ of a simple Lie triple system is nondegenerate. As mentioned in (1.4) $L$ is either simple or a direct sum of two simple ideals. Hence, it suffices to show that a simple Lie algebra defined over a field of characteristic 0 is nondegenerate. But this follows as in (1) since by [9] the ideal generated by all absolute zero divisors is locally nilpotent.

Recall from (1.1)(a) that the annihilator of an ideal $I$ of a nondegenerate Lie algebra $L$ is given by $\operatorname{Ann}_{I}(L)=\{x \in L \mid[x,[I, x]]=0\}$. The following two results give analogs of this fact for Lie triple systems and for Kantor pairs.

Theorem 3.2. Let $T$ be a nondegenerate Lie triple system and $I$ an ideal of $T$. Then $\operatorname{Ann}_{T}(I)=\{x \in T \mid[x, I, x]=0\}$.

Proof. The inclusion from left to right being obvious, let us consider $x \in T$ satisfying $[x, I, x]=0$ and put $\mathcal{I}:=[T, I] \oplus I$, the ideal of $\mathcal{L}_{T}$ generated by $I$. Then for $y, y^{\prime} \in I$ and $t \in T$ we have $\left[x,\left[x,\left[x,[y, t]+y^{\prime}\right]\right]\right]=[x,[x,[x,[y, t]]]]=[x,[x,[[x, y], t]-$ $[[x, t], y]]] \in[x,[x, I]]=0$, i.e., $x$ is an $\mathcal{I}$-Jordan element in $\mathcal{L}_{T}$. We can therefore consider the generalized Jordan algebra $\mathcal{I}_{x}$ of $\mathcal{I}$ at $x$. Because $\mathcal{L}_{T}$ is nondegenerate by (2.4), so is $\mathcal{I}$ by (1.1). Hence $\mathcal{I}_{x}$ is a nondegenerate Jordan algebra $(2.2)(3)$. Moreover, since $\mathcal{I}$ is a $\mathbb{Z}_{2}$-graded Lie algebra and $x$ is homogeneous, $\operatorname{ker}_{\mathcal{I}}(x)=\{a \in$ $\mathcal{I} \mid[x,[x, a]]=0\}$ is $\mathbb{Z}_{2}$-graded and so is then $\mathcal{I}_{x}=\mathcal{I} / \operatorname{ker}_{\mathcal{I}}(x)$. By definition of the Jordan algebra product, $\left(\mathcal{I}_{x}\right)_{0}=\{\bar{a} \mid a \in I\}$ and $\left(\mathcal{I}_{x}\right)_{1}=\{\bar{a} \mid a \in[T, I]\}$. But $\operatorname{ker}_{\mathcal{I}}(x) \cap I=I$ by assumption, so that $\left(\mathcal{I}_{x}\right)_{0}=0$. By nondegeneracy we then get $\mathcal{I}_{x}=0$, which says $\mathcal{I}=\operatorname{ker}_{\mathcal{I}}(x)$. Hence $x \in T \cap \operatorname{Ann}_{\mathcal{L}_{T}}(\mathcal{I})=\operatorname{Ann}_{T}(I)$.

From (3.2) and (1.8)(3) we now get:

Corollary 3.3. Let $V$ be a nondegenerate Kantor pair and $I=\left(I^{+}, I^{-}\right)$an ideal of $V$. Then $\operatorname{Ann}_{V}(I)^{\sigma}=\left\{x \in V^{\sigma} \mid\{x, I, x\}=0\right\}$.

An easy consequence of (3.2) and (3.3) is:

Corollary 3.4. Every ideal of a nondegenerate Kantor pair or Lie triple system is also nondegenerate.

The remaining two results deal with Kantor pairs. First, we show that nonzero ideals in nondegenerate Kantor pairs cannot have a zero side:

Proposition 3.5. Let $V$ be a nondegenerate Kantor pair and let $I=\left(I^{+}, I^{-}\right)$be an ideal of $V$ or $V^{\mathrm{op}}$. If $I^{\sigma}=0$, then $I^{-\sigma}=0$. In particular, if $x \in V^{\sigma}$ satisfies $\left\{V^{-\sigma}, x, V^{-\sigma}\right\}=0$, then $x=0$.

Proof. Let us suppose $I=\left(I^{+},\{0\}\right)$. From (2.5) we know that $\mathcal{L}_{V}$ is a nondegenerate Lie algebra. By $(2.6)(1)$, the ideal of $\mathcal{L}_{V}$ generated by $I$ is $\mathcal{I}:=$ $\left[V^{+}, I^{+}\right] \oplus I^{+} \oplus\left[V^{-}, I^{+}\right] \subset L_{2} \oplus L_{1} \oplus L_{0}$. Since $\mathcal{I}$ is nondegenerate by (1.1), $I^{+}=0$ and so $I=0$.

Lemma 3.6. Let $V$ be a Kantor pair and $I^{\sigma}$ a submodule of $V^{\sigma}$. Then $I=$ $\left(I^{\sigma},\left\{V^{-\sigma}, I^{\sigma}, V^{-\sigma}\right\}\right)$ is an ideal of $V$ or $V^{\text {op }}$ if and only if $\left\{I^{\sigma}, V^{-\sigma}, V^{\sigma}\right\}+$ $\left\{V^{\sigma}, V^{-\sigma}, I^{\sigma}\right\} \subset I^{\sigma}$.

Proof. The condition $\left\{I^{\sigma}, V^{-\sigma}, V^{\sigma}\right\}+\left\{V^{\sigma}, V^{-\sigma}, I^{\sigma}\right\} \subset I^{\sigma}$ is obviously necessary for $I$ to be an ideal. Conversely, if it is fulfilled, it suffices to verify that 
$\left\{V^{-\sigma}, V^{\sigma}, I^{-\sigma}\right\}+\left\{I^{-\sigma}, V^{\sigma}, V^{-\sigma}\right\} \subset I^{-\sigma}$ and $\left\{V^{\sigma}, I^{-\sigma}, V^{\sigma}\right\} \subset I^{\sigma}$. These three conditions are established below, where $a, c, u, v \in V^{-\sigma}, b, d \in V^{\sigma}$, and $y \in I^{\sigma}$ :

$$
\begin{aligned}
\{a, b,\{u, y, v\}\}= & \{\{a, b, u\}, y, v\}-\{u,\{b, a, y\}, v\}+\{u, y,\{a, b, v\}\} \\
& \in\left\{V^{-\sigma}, I^{\sigma}, V^{-\sigma}\right\}=I^{-\sigma}, \\
\{\{u, y, v\}, b, a\}= & \{u, y,\{v, b, a\}\}+\{v,\{y, u, b\}, a\}-\{v, b,\{u, y, a\}\} \\
& \in\left\{V^{-\sigma}, I^{\sigma}, V^{-\sigma}\right\}+\left\{V^{-\sigma}, V^{\sigma},\left\{V^{-\sigma}, I^{\sigma}, V^{-\sigma}\right\}\right\} \\
& \subset\left\{V^{-\sigma}, I^{\sigma}, V^{-\sigma}\right\}=I^{-\sigma}, \\
\{b,\{u, y, v\}, d\}= & -\{y, u,\{b, v, d\}\}+\{\{y, u, b\}, v, d\}+\{b, v,\{y, u, d\}\} \in I^{\sigma} .
\end{aligned}
$$

In the following result we characterize when Kantor pairs are Jordan pairs by building the biggest Jordan ideal of a Kantor pair. We recall that an ideal of a Kantor pair (or of any algebraic structure for that matter) is called essential if it has nonzero intersection with any nonzero ideal. In a prime Kantor pair, any non-zero ideal is essential.

Proposition 3.7. Let $V$ be a nondegenerate Kantor pair and define for $\sigma=+$ and $\sigma=-$

$$
I^{\sigma}:=\left\{x \in V^{\sigma} \mid K_{x, c} a=0=K_{a, b} x, \quad \forall c \in V^{\sigma}, a, b \in V^{-\sigma}\right\} .
$$

(1) Then $I:=\left(I^{+}, I^{-}\right)$is the biggest ideal of $V$ which is a Jordan pair with respect to the given triple product. Moreover, for $\sigma=+$ and $\sigma=-$,

$$
I^{\sigma}=\left\{x \in V^{\sigma} \mid K_{x, c}=0 \quad \forall c \in V^{\sigma}\right\} .
$$

(2) $V$ is a Jordan pair if and only if $I$ is an essential ideal, in which case $V=I$. In particular, if $V \neq 0$ is also prime, then $V$ is a Jordan pair if and only if $I \neq 0$.

Proof. (1) We first show that $\left(I^{\sigma},\left\{V^{-\sigma}, I^{\sigma}, V^{-\sigma}\right\}\right), \sigma= \pm$, are ideals of $V$ (this does not need the assumption that $V$ is nondegenerate). By (3.6) and the definition of $I^{\sigma}$ it suffices to show that $\left\{V^{\sigma}, V^{-\sigma}, I^{\sigma}\right\} \subset I^{\sigma}$. Thus, let $x \in I^{\sigma}, a, d \in V^{\sigma}$ and $b, c, e \in V^{-\sigma}$. Then

$$
\begin{aligned}
\{\{a, b, x\}, c, d\}= & \{a, b,\{x, c, d\}\}+\{x,\{b, a, c\}, d\}-\{x, c,\{a, b, d\}\} \\
= & \{a, b,\{d, c, x\}\}+\{d,\{b, a, c\}, x\}-\{\{a, b, d\}, c, x\} \\
= & \{\{a, b, d\}, c, x\}-\{d,\{b, a, c\}, x\}+\{d, c,\{a, b, x\}\} \\
& +\{d,\{b, a, c\}, x\}-\{\{a, b, d\}, c, x\}=\{d, c,\{a, b, x\}\}, \\
\{c,\{a, b, x\}, e\}- & \{e,\{a, b, x\}, c\}=K_{c, e} V_{a, b} x=-V_{b, a} K_{c, e} x+K_{K_{c, e} a, b} x=0,
\end{aligned}
$$

so $\left\{V^{\sigma}, V^{-\sigma}, I^{\sigma}\right\} \subset I^{\sigma}$. In particular, $J=\left(J^{+}, J^{-}\right)=\left(I^{+},\left\{V^{-}, I^{+}, V^{-}\right\}\right)$is an ideal of $V$. By $(2.6)(1)$,

$$
\mathcal{J}:=\left[J^{+}, V^{+}\right] \oplus J^{+} \oplus\left(\left[J^{+}, V^{-}\right]+\left[V^{+}, J^{-}\right]\right) \oplus J^{-} \oplus\left[J^{-}, V^{-}\right]
$$

is the ideal generated by $J^{+} \cup J^{-}$in $\mathcal{L}_{V}=L=L_{-2} \oplus L_{-1} \oplus L_{0} \oplus L_{1} \oplus L_{2}$. Now, given $y \in J^{+}, x \in V^{+}$and $z \in V^{-}$, we have $[[y, x], z]=[[y, z], x]-[[x, z], y]=$ $K_{y, x} z=0$, which implies that $0=\left[J^{+}, V^{+}\right]=\pi_{2}(\mathcal{J})$ where $\pi_{i}$ denotes the canonical projection onto the $L_{i}$-component of $L$. Since $\mathcal{L}_{V}$ is nondegenerate by $(2.4), 0=$ 
$\pi_{-2}(\mathcal{J})=\left[J^{-}, V^{-}\right]$, i.e., $K_{J^{-}, V^{-}} V^{+}=0$. Moreover, $L_{2} \subset \operatorname{Ann}_{\mathcal{L}_{V}}(\mathcal{J})$ by $(1.1)(\mathrm{a})$ because $\mathcal{J}$ is 3 -graded. Hence $\left[\left[V^{+}, V^{+}\right], J^{-}\right]=0$, i.e., $K_{V^{+}, V^{+}} J^{-}=0$. Therefore, $\left\{V^{-}, I^{+}, V^{-}\right\}=J^{-} \subset I^{-}$which implies that $I=\left(I^{+}, I^{-}\right)$is an ideal of $V$. Now $\mathcal{J} \subset L_{-1} \oplus L_{0} \oplus L_{1}$, and every ideal $K$ of $V$ that generates in $\mathcal{L}_{V}$ an ideal contained in $L_{1} \oplus L_{0} \oplus L_{-1}$ necessarily has $K \subset I$. Hence (a) holds.

To prove that $I$ is the biggest ideal which is a Jordan pair, let $K=\left(K^{+}, K^{-}\right)$ be an ideal of $V$ such that $K$ is a Jordan pair. Again by (2.6)(1),

$$
\mathcal{K}:=\left[K^{+}, V^{+}\right] \oplus K^{+} \oplus\left[K^{+}, V^{-}\right]+\left[V^{+}, K^{-}\right] \oplus K^{-} \oplus\left[K^{-}, V^{-}\right]
$$

is the ideal of $\mathcal{L}_{V}$ generated by $K$. We know that $\mathcal{K}$ is a nondegenerate Lie algebra. Applying (2.6)(1) one more time, it follows that the ideal of $\mathcal{K}$ generated by $K^{+} \cup K^{-}$ is

$$
\mathcal{K}^{\prime}:=\left[K^{+}, K^{+}\right] \oplus K^{+} \oplus\left[K^{+}, K^{-}\right] \oplus K^{-} \oplus\left[K^{-}, K^{-}\right] .
$$

Now, since $\mathcal{K}^{\prime}$ is nondegenerate and $\left[\left[K^{\sigma}, K^{\sigma}\right], K^{-\sigma}\right]=0$ (recall that $K$ is a Jordan pair) we have that $\left[\left[K^{\sigma}, K^{\sigma}\right], \mathcal{K}^{\prime}\right]=0$. So $\left[K^{\sigma}, K^{\sigma}\right]=0$ by nondegeneracy of $\mathcal{K}^{\prime}$, and this implies, viewing $\left[K^{\sigma}, K^{\sigma}\right]$ as elements of $\mathcal{L}_{V}$, that $\left[\left[K^{\sigma}, K^{\sigma}\right], V^{-\sigma}\right]=0$ and $K_{y, y^{\prime}} b=0$ for every $y, y \in K^{\sigma}$ and $b \in V^{-\sigma}$. Moreover, $\left[K^{\sigma}, K^{\sigma}\right]=0$ implies that $\mathcal{K}^{\prime}$ is 3 -graded and then by $(1.1)\left(\right.$ a) that $\left[V^{\sigma}, K^{\sigma}\right] \subset \operatorname{Ann}_{\mathcal{K}}\left(\mathcal{K}^{\prime}\right)$. In particular, $K_{y, a} y^{\prime}=0$ for every $y \in K^{\sigma}, a \in V^{\sigma}$ and $y^{\prime} \in K^{-\sigma}$. Finally, for every $y \in K^{\sigma}, y^{\prime} \in K^{-\sigma}, a \in V^{\sigma}$ and $b \in V^{-\sigma}$ we have:

$$
\left\{K_{y, a} b, y^{\prime}, K_{y, a} b\right\}=V_{K_{y, a} b, y^{\prime}} K_{y, a} b=-K_{y, a} V_{y^{\prime}, K_{y, a} b} b+K_{K_{y, a} y^{\prime}, K_{y, a} b} b=0
$$

and, since $\mathcal{K}^{\prime}$ is nondegenerate, $K_{y, a} b=0$ for every $y \in K^{\sigma}, a \in V^{\sigma}$ and $b \in V^{-\sigma}$. Thus, by (1), $K \subset I$.

(2) Let $\mathcal{I}=I^{+} \oplus\left(\left[I^{+}, V^{-}\right]+\left[V^{+}, I^{-}\right]\right) \oplus I^{-}$be the ideal of $\mathcal{L}_{V}$ generated by $I^{+} \cup I^{-}$, and notice that $L_{2} \cup L_{-2} \subset \operatorname{Ann}_{\mathcal{L}_{V}}(\mathcal{I})=0$ and $\mathcal{I} \subset L_{1} \oplus L_{0} \oplus L_{-1}$. Now, $I$ is an essential ideal of $V$ if and only if $\mathcal{I}$ is an essential ideal of $\mathcal{L}_{V}$. Since the annihilator of an essential ideal is zero, we get $L_{2}=0=L_{-2}$. The converse is obvious.

Acknowledgements. The authors are grateful to B. Allison and O. Smirnov for the permission to include their results 2.6 and 2.7(3) in this paper and for helpful comments on an earlier version of this paper.

\section{REFERENCES}

1. Bruce N. Allison and John R. Faulkner, Elementary groups and invertibility for Kantor pairs, Comm. Algebra 27 (1999), no. 2, 519-556. MR 1671930 (99k:17002)

2. Bruce N. Allison and Oleg Smirnov, private communication.

3. Georgia Benkart and Oleg Smirnov, Lie algebras graded by the root system $\mathrm{BC}_{1}$, J. Lie Theory 13 (2003), no. 1, 91-132.

4. A. J. Calderón Martín and M. Forero Piulestán, Inheritance of primeness by ideals in Lie triple systems, Algebras, rings and their representations, World Sci. Publ., Hackensack, NJ, 2006, pp. 7-16. MR 2234296 (2007b:17002)

5. Antonio Fernández López, Esther García, and Miguel Gómez Lozano, The Jordan socle and finitary Lie algebras, J. Algebra 280 (2004), no. 2, 635-654. MR 2089256 (2005i:17007)

6. The Jordan algebras of a Lie algebra, J. Algebra 308 (2007), no. 1, 164-177. MR 2290916 (2007k:17049)

7. Esther García and Miguel Gómez Lozano, Jordan systems of Martindale-like quotients, J. Pure Appl. Algebra 194 (2004), no. 1-2, 127-145. MR 2086077 (2005g:17046) 
8. __ An elemental characterization of strong primeness in Lie algebras, J. Algebra 312 (2007), no. 1, 132-141. MR 2320450

9. A. N. Grishkov, Local nilpotency of an ideal of a Lie algebra generated by second-order elements, Sibirsk. Mat. Zh. 23 (1982), no. 1, 181-182, 222. MR 651890 (83d:17007)

10. Nathan Jacobson, Structure and representations of Jordan algebras, American Mathematical Society Colloquium Publications, Vol. XXXIX, American Mathematical Society, Providence, R.I., 1968. MR 0251099 (40 \#4330)

11. I. L. Kantor, Certain generalizations of Jordan algebras, Trudy Sem. Vektor. Tenzor. Anal. 16 (1972), 407-499.

12. _ Models of the exceptional Lie algebras, Dokl. Akad. Nauk SSSR 208 (1973), 12761279 .

13. William G. Lister, A structure theory of Lie triple systems, Trans. Amer. Math. Soc. 72 (1952), 217-242. MR 0045702 (13,619d)

14. Ottmar Loos, Jordan pairs, Springer-Verlag, Berlin, 1975, Lecture Notes in Mathematics, Vol. 460. MR 0444721 (56 \#3071)

15. Kurt Meyberg, Lectures on algebras and triple systems, The University of Virginia, Charlottesville, Va., 1972, Notes on a course of lectures given during the academic year 1971-1972. MR 0340353 (49 \#5108)

16. Oleg N. Smirnov, Simple associative algebras with finite Z-grading, J. Algebra 196 (1997), no. 1, 171-184. MR 1474168 (98i:16045)

17. E. I. Zelmanov, Absolute zero-divisors in Jordan pairs and Lie algebras, Mat. Sb. (N.S.) 112(154) (1980), no. 4(8), 611-629. MR 587040 (82b:17010)

18. _ Lie algebras with algebraic associated representation, Mat. Sb. (N.S.) 121(163) (1983), no. 4, 545-561. MR 716113 (84k:17006)

Departamento de Matemática Aplicada, Universidad Rey Juan Carlos, 28933 Móstoles (MADRID), SPAIN

E-mail address: esther.garcia@urjc.es

Departamento de Álgebra, Geometría y Topología, Universidad de Málaga, 29071 MÁLAGA, SPAIN

E-mail address: magomez@agt.cie.uma.es

Department of Mathematics and Statistics, University of Ottawa, Ontario K1N 6N5, CANADA

E-mail address: neher@uottawa.ca 